\title{
Broken rotor bar fault diagnostic for DTC fed induction motor using stator instantaneous complex apparent power envelope signature analysis
}

\author{
Lachtar Salah', Ghoggal Adel $^{2}$, Koussa Khaled ${ }^{3}$, Bouraiou Ahmed ${ }^{4}$, Attoui Issam ${ }^{5}$ \\ ${ }^{1,2}$ LGEB- laboratory, Department of Electrical Engineering, University of Biskra, Algeria \\ 1,3,4 Unité de recherche en Energie Renouvelables en milieu saharien, Centre de Développement des Energies \\ Renouvelables, Algeria \\ ${ }^{3}$ Department of Electrical Engineering, University of Bechar, Algeria \\ ${ }^{5}$ Research Center in Industrial Technologies CRTI, Algeria
}

\section{Article Info \\ Article history: \\ Received Dec 9, 2018 \\ Revised Feb 12, 2019 \\ Keywords: \\ Broken rotor bar \\ DTC \\ Fault diagnosis \\ SCIM \\ SICAPE \\ Signature analysis}

Accepted Mar 20, 2019

\begin{abstract}
The broken rotor bar is an unexpected fault and a common cause of induction motor failures that threaten the structural integrity of electric machines. In this paper, a new approach to a broken rotor bar diagnosis, without slip estimation, based on the envelope of the stator instantaneous complex apparent power (SICAP) is proposed. The envelope is obtained from the SICAP modulation and then transferred to a computer for monitoring the characteristic frequency and its amplitude using the Fast Fourier Transform (FFT). For this purpose, the winding function approach (WFA) is used to simulate the broken rotor bar occurrence in a squirrel cage induction motor (SCIM) fed on direct torque control (DTC). The obtained simulation results confirm the interest and efficiency of the proposed technique. Even when the induction motor is operating at the no-load level condition, the proposed method is also efficient to detect the broken rotor bar fault at low slip
\end{abstract}

Copyright $(2019$ Institute of Advanced Engineering and Science. All rights reserved.

\section{Corresponding Author:}

Lachtar Salah,

LGEB- laboratory, Department of Electrical Engineering,

University of Biskra,

BP 145 RP Biskra 07000, Algeria.

Email: lachrtarsalahba@gmail.com

\section{INTRODUCTION}

Induction motors are widely used in automated production systems. Most of these systems require high performances control to maintain a stable velocity independent of any load disturbances or model uncertainties. The SCIM has proven itself through the success that prevails in the field of speed variation and through an influx of research work which is exceptionally intended [1,2]. The particular reasons for this acquired confidence in the SCIM are based on its intrinsic qualities such as its simplicity of construction, its mechanical robustness and low purchase and manufacturing costs.

However, asynchronous machines are often subjected during their operation to several stresses of different natures (excessive heating, magnetic weariness caused by the electromagnetic forces and environmental stresses that the rotor must undergo during its usual use). The accumulation of these constraints causes defects in the different parts of the machine such as stator short-circuits [3], eccentricity [4], broken rotor bar [5], which generates ultimately stops, leading to production losses. To avoid these unscheduled shutdowns, the researchers haves been working for several years to develop maintenance techniques.

It is well known that the widespread method to detect broken rotor bars is motor current signature analysis (MCSA), which is based on signal processing tools such as Fast Fourier Transforms (FFT). It 
depends on detecting the sidebands $(1 \pm 2 \mathrm{ks}) \mathrm{f}$ ( $\mathrm{s}$ is the rotor slip, $\mathrm{f}$ is the fundamental frequency and $\mathrm{k}=1,2$, $3 . .$.$) , inducing in the stator current spectrum producing by geometric and magnetic unbalances caused by$ broken rotor bars. Therefore, these sideband frequencies appearing around the fundamental frequency and the evaluation of their amplitudes can be identify and used as a reliable and accurate approach to diagnose rotor bar faults.

However, the MCSA has some drawbacks, where the reliability and the accuracy are affected by the diagnosis of rotor asymmetries at very low slip. Therefore, the sideband frequencies become quite close to the fundamental frequency [6-8]. In addition, in the closed-loop drives, the controller loop compensates the fault effect i.e. mask the fault effect $[9,10]$. Therefore, using stator current analysis causes a difficulty in fault detection, which motivated researchers attempted to use other quantities for fault analysis [11-16].

To handle these problems, other methods based on the amplitude modulation of the three-phase stator current induced by a broken rotor bar is used in aid of diagnostic. In fact, the rotor fault effect can be localized in the stator current envelope spectrum at frequencies expressed by the components $2 \mathrm{ksf}$ [17-20].

This paper addresses these difficulties with an innovative method based on the amplitude modulation of the SICAP is the so-called envelope (SICAPE) to detect the broken rotor bars fault in a DTCfed induction motor. A second-order low-pass filter is used to eliminate the high frequency generated by the DTC inverter. The Fast Fourier Transform (FFT) is applied to the filtered envelope component in order to extract the fault indexes using the normalized band amplitude of the spectrum components. The simulation is performed using Matlab/Simulink. The obtained results show that the proposed method is able to detect the broken rotor bars fault at any load level condition in the SCIM drives as well as at very low slip condition.

\section{MATERIAL AND METHODS}

The broken bar fault in the SCIM is modeled based on the winding function approach WFA. All the space harmonics in the machine are taken into account. The WFA approach predicts the performances of the differential equations model. This model refers to the coupled magnetic approach by treating the current in each rotor bar as an independent variable [21]. The induction motor model is considered with the following simplifying assumptions [22].

- The magnetic circuits are unsaturated,

- The inter-bar current is neglected,

- The skin effects are neglected,

- The effect of slots is neglected,

- The distribution of the magnetomotive force in the air-gap is sinusoidal.

The rotor squirrel-cage modeling is based on the equivalent diagram of $(\mathrm{Nr}+1)$ meshes as shown in Figure 1. Each mesh is substituted by an equivalent circuit represented by a rotor bar and a segment of endring, respectively under resistive and inductive nature $(\mathrm{Rb}, \mathrm{Re}, \mathrm{Lb}, \mathrm{Le})$.

$\mathrm{Rb}$ and $\mathrm{Lb}$, epresent the rotor bar resistance and is inductance. Re and Le are the end-ring segment resistance and its inductance. The rotor voltage equations of the $\mathrm{Nr}$ loops can be expressed as follow

$$
\left(2 R_{e k}+R_{b(k+1)}+R_{b k}\right) \cdot I_{r k}-R_{b k} \cdot I_{r(k-1)}-R_{b(k+1)} \cdot I_{r(k+1)}-R_{e} I_{e\left(N_{r}+1\right)}+\frac{d \phi_{r k}}{d t}=0(1)
$$

Where, $\phi \mathrm{r}, \mathrm{k}$ is the rotor flux crossing the rotor loop $\mathrm{k}$.

The dynamic mathematical model of the induction motor can be written in vector matrix as

$$
[\mathrm{V}]=[\mathrm{R}] \cdot[\mathrm{I}]+\frac{d}{d t}([L][I])
$$

Where the voltage vector matrix [V] is given by

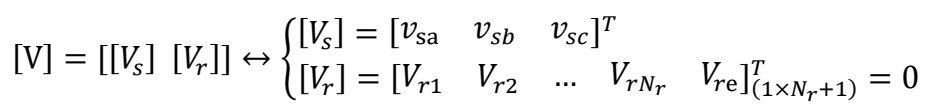

Where $N r$ is the number of rotor bars.

Int J Pow Elec \& Dri Syst, Vol. 10, No. 3, Sep 2019 : 1187 - 1196 


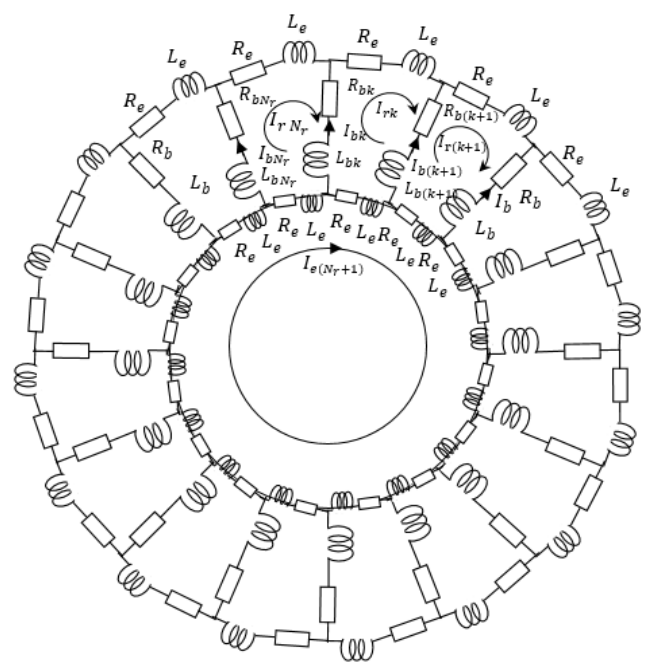

Figure 1. The equivalent circuit of the rotor squirrel-cage

The current vector matrix $[\mathrm{I}]$ is composed

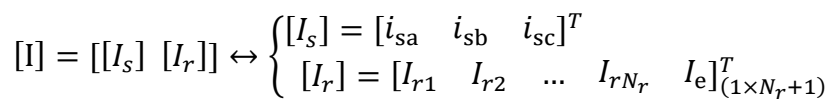

The global resistance matrix $[R]$ is given as follow

$$
[R]=\left[\begin{array}{cc}
{\left[R_{s}\right]} & {[0]_{\left(3, N_{r}+1\right)}} \\
{[0]_{\left(3, N_{r}+1\right)}} & {\left[R_{r}\right]}
\end{array}\right]
$$

Where, $\left[R_{s}\right]$ is the diagonal matrix of the stator resistances phases of $(m, m)$ dimensions.

$$
\left[R_{s}\right]=\left[\begin{array}{ccc}
R_{s 1} & 0 & 0 \\
0 & R_{s 2} & 0 \\
0 & 0 & R_{s 3}
\end{array}\right]
$$

$R_{s 1}, R_{s 2}$ and $R_{s 3}$ are the identical resistances of stator phases winding. The resistance $\left[R_{r}\right]$ is a symmetric matrix $(\mathrm{Nr}+1, \mathrm{Nr}+1)$ given as

$$
\left[R_{r}\right]=\left[\begin{array}{cccccc}
R_{r r} & -R_{b} & 0 & 0 & -R_{b\left(1, N_{r}\right)} & -R_{e\left(1, N_{r}+1\right)} \\
-R_{b} & R_{r r} & -R_{b} & \vdots & 0 & -R_{e} \\
0 & -R_{b} & \mathrm{R}_{r r} & -R_{b} & \vdots & \vdots \\
\vdots & 0 & -R_{b} & \ddots & -R_{b} & \vdots \\
-R_{b\left(N_{r}, 1\right)} & \vdots & 0 & -R_{b} & \mathrm{R}_{r r\left(N_{r}, N_{r}\right)} & \vdots \\
-R_{e\left(N_{r}+1,1\right)} & -R_{e} & -R_{e} & -R_{e} & -R_{e} & -R_{e\left(N_{r}+1, N_{r}+1\right)}
\end{array}\right]
$$

Where, $R_{r r}=2\left(R_{b}+R_{e}\right), R_{b}$ is the rotor bar resistance and $R_{e}$ is the end ring resistance. The global inductance matrix $[L]$ is as follow

$$
[L]=\left[\begin{array}{cc}
{\left[L_{s}\right]} & {\left[M_{s r}\right]_{\left(3, N_{r}+1\right)}} \\
{\left[M_{r s}\right]_{\left(N_{r}+1,3\right)}} & {\left[L_{r}\right]_{\left(N_{r}+1, N_{r}+1\right)}}
\end{array}\right]
$$

Where the inductance matrix $\left[L_{s}\right]$ is given by 


$$
\left[L_{s}\right]=\left[\begin{array}{ccc}
L_{s 1} & M_{s} & M_{s} \\
M_{s} & L_{s 2} & M_{s} \\
M_{s} & 0 & L_{s 3}
\end{array}\right]
$$

Where, $L_{s 1}, L_{s 2}$ and $L_{s 3}$ are the identical proper inductances of the stator coil and $M_{s}$ is the mutual inductance of the stator phases. The inductance matrix $\left[L_{r}\right]$ is a symmetric matrix $\left(N_{r}+1, N_{r}+1\right)$ given by

$$
\left[L_{r}\right]=\left[\begin{array}{cccccc}
L_{r r} & \left(M_{r r}-L_{b}\right) & M_{r r} & \ldots & \left(M_{r r}-L_{b}\right) & -L_{e\left(1, N_{r}+1\right)} \\
\left(M_{r r}-L_{b}\right) & L_{r r} & \left(M_{r r}-L_{b}\right) & M_{r r} & M_{r r} & -L_{e} \\
M_{r r} & \left(M_{r r}-L_{b}\right) & L_{r r} & \left(M_{r r}-L_{b}\right) & \vdots & \vdots \\
\vdots & M_{r r} & \left(M_{r r}-L_{b}\right) & \ddots & \left(M_{r r}-L_{b}\right) & \vdots \\
\left(M_{r r}-L_{b}\right) & \vdots & M_{r r} & \left(M_{r r}-L_{b}\right) & L_{r r}\left(N_{r}, N_{r}\right) & \vdots \\
-L_{e\left(N_{r}+1,1\right)} & -L_{e} & -L_{e} & -L_{e} & -L_{e} & -L_{e\left(N_{r}+1, N_{r}+1\right)}
\end{array}\right]
$$

Where, $L_{r r}=L_{m r}+2\left(L_{b}+L_{e}\right), L_{m r}$ is the magnetizing inductance of each rotor loop. $L_{b}$ is the rotor bar leakage inductance, $L_{e}$ is the rotor end ring leakage inductance and $M_{r r}$ is the mutual inductance between two rotor loops.

The mutual inductance matrix $\left[M_{s r}\right]$, between the stator windings and rotor loops of $\left(3, N_{r}+\right.$ 1) dimensions, which signified that the vector of the rotor currents comprise $(k+1)$ elements corresponding to the number of rotor cage bars $\left(N_{r}\right)$, plus the end-ring loop $\left(N_{r}+1\right)$.

$$
\left[M_{s r}\right]=\left[\begin{array}{lllll}
M_{s 1 r k} & M_{s 1 r k+1} & . & . & M_{s 1 r N_{r}+1} \\
M_{s 2 r 2 k} & M_{s 2 r k+1} & . & . & M_{s 2 r N_{r}+1} \\
M_{s 3 r 3 k} & M_{s 3 r k+1} & . & . & M_{s 3 r N_{r}+1}
\end{array}\right]
$$

The mechanical rotor equations of velocity $\Omega_{r}$ and position $\theta_{r}$ are represented as follows

$$
\left\{\begin{array}{l}
J \frac{d \Omega_{r}}{d t}=T_{e}-f_{v} \cdot \Omega_{r}-T_{L} \\
\frac{d \theta_{r}}{d t}=\Omega_{r}
\end{array}\right.
$$

Where, $J$ is the moment of inertia, $T_{L}$ is the load torque and $T_{e}$ the electromagnetic torque produced by the machine is obtained by

$$
T_{e}=0,5 \cdot[I]^{T} \frac{d\left[L\left(\theta_{r}\right)\right]}{d \theta_{r}}[I]
$$

In order to simulate the broken rotor bars failure using MATLAB software, the broken bar resistance $R_{b}$ is strongly increased by an additional resistance $R_{b F}$ called the defect resistance, expressed by the following matrix.

$$
\left[R_{b F}\right]=\left[\begin{array}{cccccc}
0 & 0 & 0 & \ldots & \ldots & 0 \\
0 & 0 & 0 & 0 & \ldots & 0 \\
0 & 0 & R_{b F k} & -R_{b F k} & 0 & \vdots \\
\vdots & 0 & -R_{b F k} & R_{b F k} & 0 & \vdots \\
\vdots & \vdots & 0 & 0 & \vdots & \vdots \\
\vdots & \vdots & \vdots & \vdots & \vdots & \vdots
\end{array}\right]
$$

The resistance matrix $\left[R_{r}\right]$ of the rotor squirrel cage is changed taking into account the additional defect resistance matrix $\left[R_{b F}\right]$, the new resistance matrix $\left[R_{r d}\right]$ is defined as follow

$$
\left[R_{r d}=\left[R_{r}\right]+\left[R_{b F}\right]\right]
$$

\section{DIAGNOSIS OF ROTOR FAILURE IN DTC FED INDUCTION MOTOR}

DTC is an AC drive technology that directly controls the torque and magnetizing flux of the motor (Figure 2). This method estimates the magnetic flux and the motor torque by measuring the supplied voltages 
and currents. If the torque or flux falls outside a predefined tolerance range, the inverter transistors are switched to the next state so that they return to the range as fast as possible [23].

\subsection{Stator flux behavior}

In the $(\alpha, \beta)$ reference, the stator flux can be obtained by the following equation

$$
\bar{\varphi}_{s}=\int_{0}^{t}\left(\bar{v}_{s}-R_{s} \bar{l}_{s}\right) d t
$$

Where, $\bar{\varphi}_{s}$ is the stator flux vector, $\bar{\varphi}_{s 0}$ is the initial stator flux vector of $\bar{\varphi}_{s}$ and, $R_{s}$ is the stator resistance. If the voltage drop due to the stator resistance neglected, $\bar{\varphi}_{s}$ can be written as follow

$$
\bar{\varphi}_{s}=\bar{\varphi}_{s 0}+\int_{0}^{t} \bar{v}_{s} d t
$$

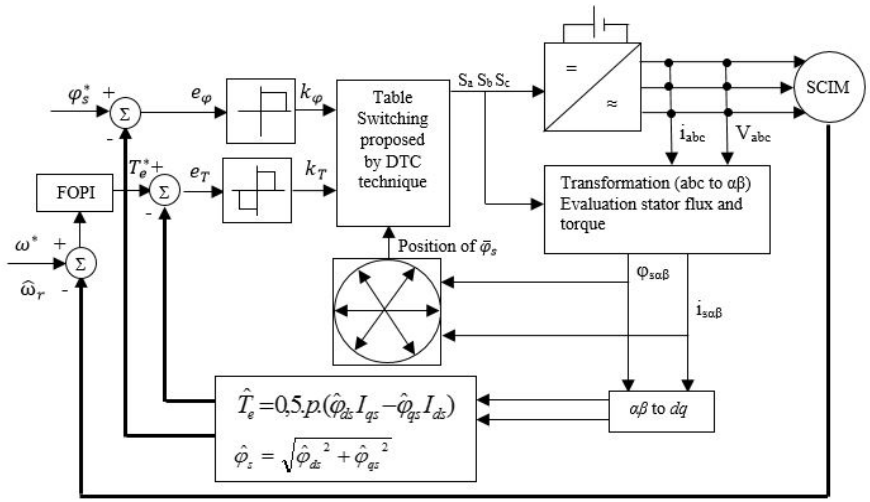

Figure 2. DTC block diagram for induction motor drive.

The estimated flux is carried out by the integration of the stator voltage. The selection of the voltage vector depends on the position of stator flux.

$$
\hat{\varphi}_{s}=\sqrt{\hat{\varphi}_{s \alpha}^{2}+\hat{\varphi}_{s \beta}^{2}}
$$

\subsection{Torque behavior}

The electromagnetic torque component is proportional to the vector product between the stator and rotor flux $\bar{\varphi}_{s}$ and $\bar{\varphi}_{r}$. The electromagnetic torque equation is giving by

$$
T_{e}=\frac{1}{2} P \bar{l}_{s} j \bar{\varphi}_{s}
$$

\subsection{Switching strategy development}

Based on the hysteresis state of the torque, the flux and the switching sector of the stator flux, which is designated by $\alpha$ (20), the DTC algorithm (Figure 2) selects the suitable inverter voltage vector to apply to the induction motor from the Table 1 . The outputs of the switching vectors in the different stator flux sectors are parameters for the inverter switching devices.

Where,

$$
\alpha=\angle \bar{\varphi}_{s}=\tan ^{-1}\left(\frac{\varphi_{\beta s}}{\varphi_{\alpha s}}\right)
$$

The active switching vectors are $\bar{V}_{1} \rightarrow \bar{V}_{6}$, and the zero switching vectors are $\bar{V}_{0}, \bar{V}_{7}$ as shown in the Table 1 . 


\begin{tabular}{|c|c|c|c|c|c|c|c|}
\hline & (S) & 1 & 2 & 3 & 4 & 5 & 6 \\
\hline \multirow{3}{*}{$\mathrm{k}_{\varphi=1}$} & $\mathrm{k}_{\mathrm{T}=1}$ & 110 & 010 & 011 & 001 & 101 & 100 \\
\hline & $\mathrm{k}_{\mathrm{T}=0}$ & 111 & 000 & 111 & 000 & 111 & 000 \\
\hline & $\mathrm{k}_{\mathrm{T}=-1}$ & 101 & 100 & 110 & 010 & 011 & 001 \\
\hline \multirow{3}{*}{$\mathrm{k}_{\varphi=0}$} & $\mathrm{k}_{\mathrm{T}=1}$ & 010 & 011 & 001 & 101 & 100 & 110 \\
\hline & $\mathrm{k}_{\mathrm{T}=0}$ & 000 & 111 & 000 & 111 & 000 & 111 \\
\hline & $\mathrm{k}_{\mathrm{T}=-1}$ & 001 & 101 & 100 & 110 & 010 & 011 \\
\hline
\end{tabular}

\subsection{Description of SICAP for both healthy and faulty cases}

In this section, a theoretical study from which explain the effect of broken rotor bars in the DTC fed SCIM. In the healthy case, the SICAPE determined from the rms values of current and voltage must be constant and contained only the dc component. These rms values of current and voltage provided by the DTC fed induction motor considered balanced and sinusoidal [24]. The SICAPE resulting is given by

$$
s=\|\bar{s}\|
$$

where,

$$
\begin{aligned}
& \bar{s}(t)=\sqrt{3} \cdot \bar{v}_{s} \cdot \bar{l}_{s}^{*} \\
& \bar{v}_{s}=V_{\alpha}+j V_{\beta}=\sqrt{\frac{2}{3}} V_{d c}\left(S_{a}+S_{b} e^{j \frac{2 \pi}{3}}+S_{c} e^{-j \frac{2 \pi}{3}}\right)
\end{aligned}
$$

and

$$
\bar{\iota}_{s}=I_{s \alpha}+j I_{s \beta}=\sqrt{\frac{2}{3}} I_{d c}\left(S_{a}+S_{b} e^{j \frac{2 \pi}{3}}+S_{c} e^{-j \frac{2 \pi}{3}}\right)
$$

However, when a broken rotor bar fault appears, an additional ripple component produces manifesting on the cyclically repeating envelope (Figure 5) at a rate equal to twice the slip frequency $f_{d k}=2 s f$. The apparent power $\bar{s}(t)$ becomes

$$
\bar{s}(t)=\bar{s}_{0}+\sum_{k=1}^{\infty} \bar{s}_{k} \cdot \cos \left(2 \pi f_{d k} \cdot t \pm \alpha_{s k}\right)
$$

Where, $\bar{s}_{0}$ is the component in the healthy case, and the second term in (25) is called $\bar{s}_{f}(t)$, represents the additional ripple component besides the dc component, which presents the broken rotor bar fault at the disturbance frequency $f_{d k}[25]$.

$$
\left\{\begin{array}{l}
\bar{s}_{f}(t)=\sum_{k=1}^{\infty} \bar{s}_{k} \cdot \cos \left(2 \pi f_{d k} \cdot t \pm \alpha_{s k}\right) \neq 0 \text { for } k=1 \\
\bar{s}_{f}(t)=\sum_{k=1}^{\infty} \bar{s}_{k} \cdot \cos \left(2 \pi f_{d k} \cdot t \pm \alpha_{s k}\right) \approx 0 \text { for } k \neq 1
\end{array}\right.
$$

\section{RESULTS AND DISCUSSION}

The procedures for obtaining the SICAPE devoted to signatures analysis revealing the broken rotor bars can be summarized as follows:

In the first step, a modulation of the stator apparent power is necessary to obtain the so-called envelope (Figure 3(a) and 3(b)). This envelope resulting from the modulation of the stator apparent power for a DTC fed IM in the healthy and faulty cases with one, two and three broken bars under different load conditions is shown in the obtained results presented in Figure 5. The second step, the identification of the envelope consists of extracting only the positive peak of the stator apparent power (Figure 4(a) and 4(b)). The third step, a Low-Pass Filter for the DTC fed IM. The stator apparent power, which comprises the threephase stator currents (22), which comprises a high-frequency component due to the frequency of the space vector modulation (SVM) of the DTC inverter. As a general rule, the fundamental frequency of the stator current varies from 0 to $50 \mathrm{~Hz}$ and the sampling frequency in our case went up to $4 \mathrm{kHz}$, according to the hysteresis bands of flux and torque. This SVM component is removed from the stator apparent power signal by a second-order low-pass filter. As a result, the envelope is isolated from the apparent power without a significant SVM component. Therefore, the filtered envelope of the stator apparent power is useful as an

Int J Pow Elec \& Dri Syst, Vol. 10, No. 3, Sep 2019 : 1187 - 1196 
indicator to detect broken rotor bars fault. A fast Fourier transform (FFT) is applied on the filtered envelope to detect an eventual broken rotor bars fault from which the slip frequencies appear at $2 k s f$ as shown in Figure 6.

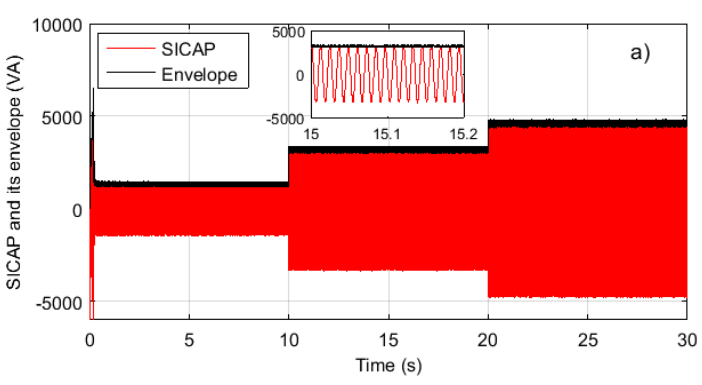

(a)

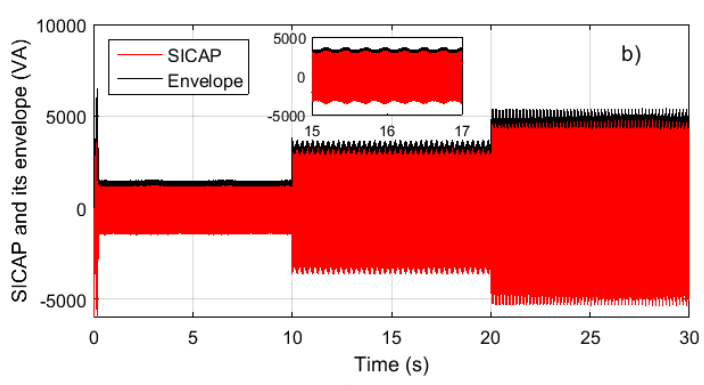

(b)

Figure 3. SICAP and its envelope for healthy case (a) and faulty case (b), under different load conditions

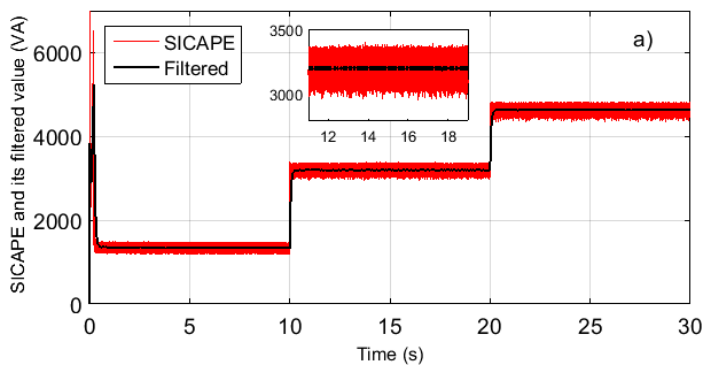

(a)

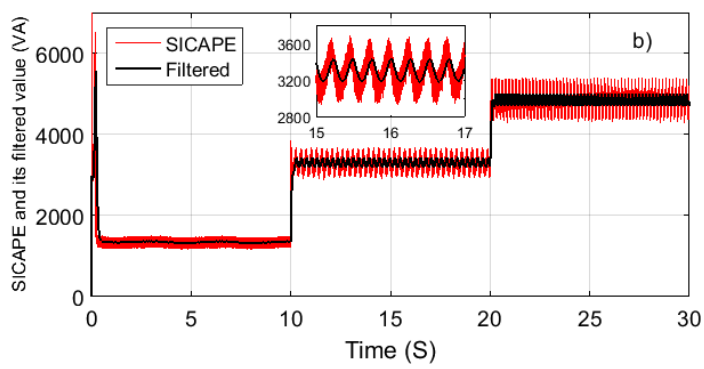

(b)

Figure 4. SICAPE and its filtered spectrum for healthy case (a) and faulty case (b), under different load conditions

Figure 5 shows the envelope and its filtered spectrum in both healthy and faulty cases divided according to the load level conditions. The simulation shows clearly in the healthy case, the filtered envelope is presented only the continuous component under no load and medium load conditions (Figure 5(a) and 5(d)). On the other hand, in the faulty case, the amplitude modulation induced by broken rotor bars fault appears clearly at no load condition (Figure 5(b) and 5(c)) and the amplitude modulation increased caused by medium load condition (Figure 5(e) and 5(f)).

Figure 6 presents the spectrum of the filtered SICAPE. In the healthy case, Figure 6(a) and 6(d) show that there is no characteristic frequency $f_{d k}$ for both no load and medium load conditions. In contrast, Figure $6(\mathrm{~b})$ shows the envelope FFT is revealed clearly the characteristic frequency $f_{d k}$ with a slight amplitude indicating the presence of a broken rotor bar fault in spite of no-load condition (very low slip). The $f_{d k}$ amplitude is increased either by the broken rotor bars number as shown in Figure 6(b) and 6(c), by the load ratio as shown in Figure 6(b) and 6(e), or by the both together as shown in Figure 6(b) and 6(f).

The investigation on the low-frequency components at $2 k s f$ makes it possible that the sampling rate of the filtered SICAPE can be decimated before FFT is performed, which greatly reduces the calculation time. Therefore, this method proved its effectiveness and reliability in simplifying the task of accurate detection of broken rotor bars under different load conditions even at no-load condition. Instead of the classical MCSA method that has demonstrated its inability to detect broken rotor bars at very low slip, and fails in detecting faults of closed loop applications $[6,10]$. 


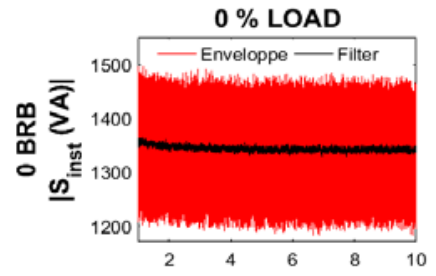

(a)

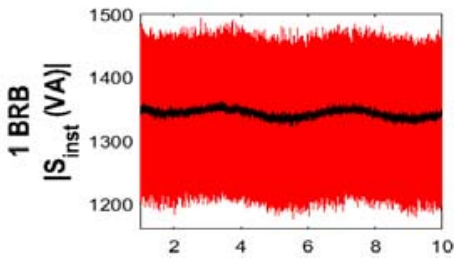

(b)

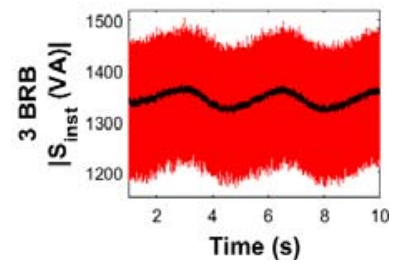

(c)

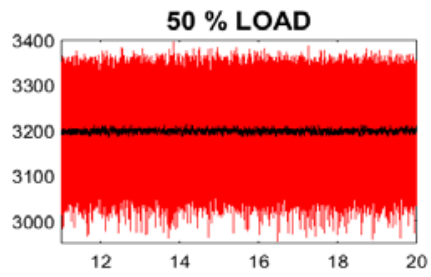

(d)



(e)

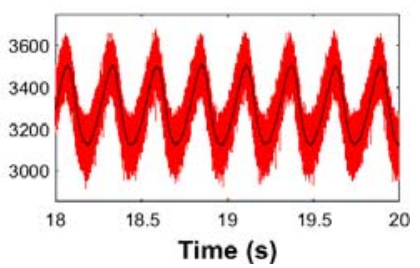

(f)

Figure 5. SICAPE and its filtered spectrum. No load condition: a) healthy case, b) 1BRB, c) 3 BRB. Medium load condition: d) healthy case, e) 1 BRB, f) 3 BRB
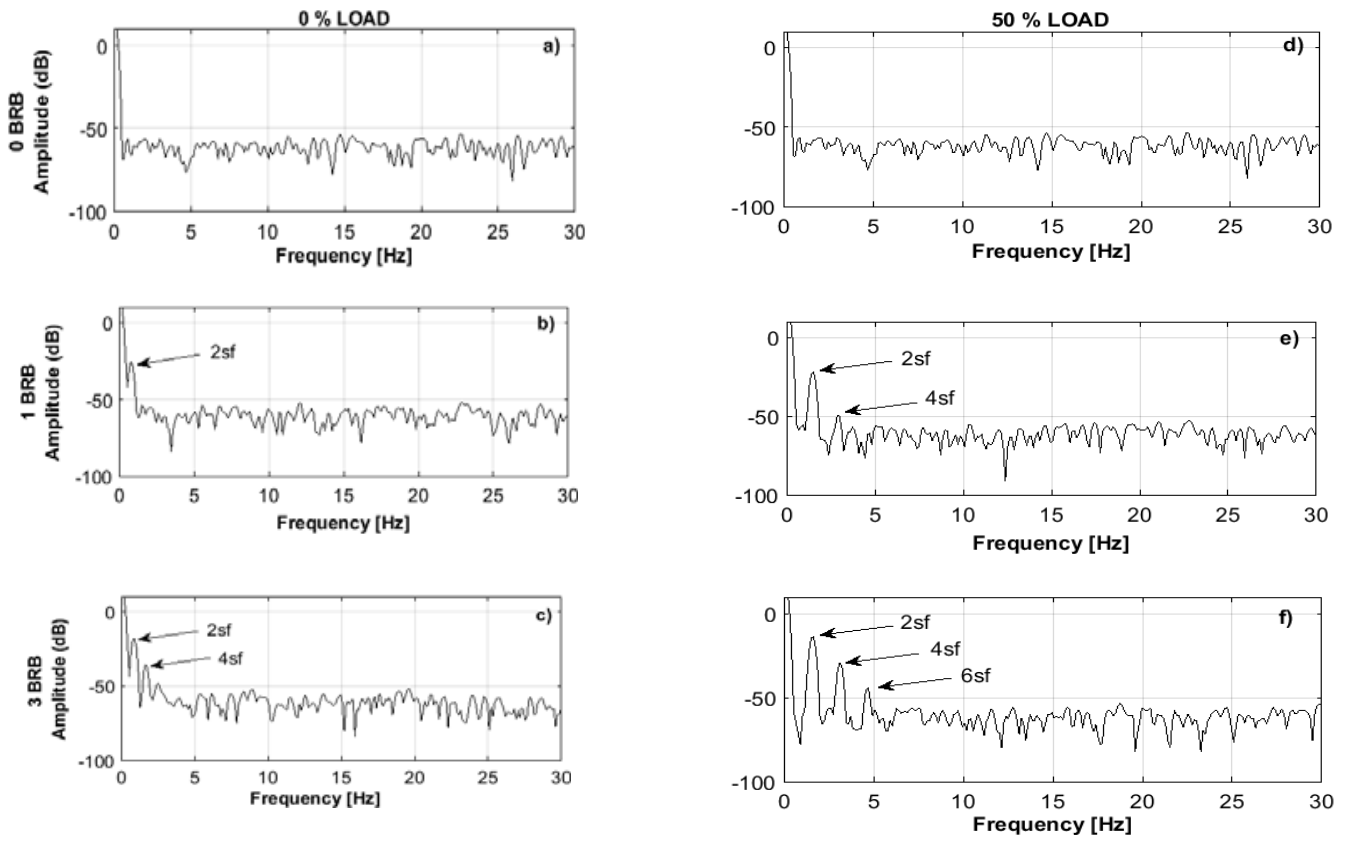

Figure 6. FFT of SICAPE filtered spectrum. No load condition: a) healthy case, b) 1BRB, c) 3 BRB. Medium load condition: d) healthy case, e) 1 BRB, f) 3 BRB 


\section{CONCLUSION}

In the scope of this work, a new approach was proposed to diagnosis the broken rotor bars fault in a DTC-fed induction motor using the signature analysis of the SICAP envelope (SICAPE). The proposed method was performed to detect the broken rotor bars fault for different load levels in the SCIM drives as well as for no-load condition. The system model was developed and implemented in Matlab/Simulink environment. The simulation was carried out in order to estimate the SCIM performances under the effect of the broken rotor bar. Based on the obtained results, the following conclusions can be drawn; first, the closedloop has no influence on the fault inductor, despite the elimination of ripples on the output quantities ensured by the effect of the DTC control. Second, the proposed method allows generating broken rotor bars fault signatures using FFT, avoiding the digital methods, which require an expensive technology. Third, the analyzed component shows only the signatures of a broken rotor bar, which makes it possible to avoid incorrectly attributed to the others electrical or mechanical defects (stator or eccentricity faults). Fourth, the proposed method is able to detect the broken rotor bar at the no-load condition and the characteristic frequency is clearly visible with a low amplitude. Lastly, a fault severity ratio is defined by the amplitude of the characteristic frequency component, which considered as a good indicator of the state of the SCIM related to the number of broken rotor bars and load level condition.

\section{REFERENCES}

[1] M. Zair and A. Hazzab, "MRAS Speed Sensorless Vector Control of Induction Motor Drives Using Predictive Adaptation Mechanism," International Journal of Power Electronics Drive Systems, vol. 9, no. 4, pp. 1523-1533, 2018.

[2] M. Es-saadi, M. Khafallah, H. Chaikhy, and H. Chaikhy, "Using the Five-Level NPC Inverter to Improve the FOC Control of the Asynchronous Machine," International Journal of Power Electronics Drive Systems, vol. 9, no. 4, pp. 1457-1466, 2018.

[3] M. Drif and A. J. M. Cardoso, "Stator fault diagnostics in squirrel cage three-phase induction motor drives using the instantaneous active and reactive power signature analyses," IEEE Trans. Ind. Informatics, vol. 10, no. 2, pp. 1348-1360, 2014.

[4] M. Akar, "Detection of a static eccentricity fault in a closed loop driven induction motor by using the angular domain order tracking analysis method," Mech. Syst. Signal Process., vol. 34, no. 1-2, pp. 173-182, 2013.

[5] R. J. Romero-Troncoso, A. Garcia-Perez, D. Morinigo-Sotelo, O. Duque-Perez, R. A. Osornio-Rios, and M. A. Ibarra-Manzano, "Rotor unbalance and broken rotor bar detection in inverter-fed induction motors at start-up and steady-state regimes by high-resolution spectral analysis," Electr. Power Syst. Res., vol. 133, pp. 142-148, 2016.

[6] R. Puche-Panadero, M. Pineda-Sanchez, M. Riera-Guasp, J. Roger-Folch, E. Hurtado-Perez, and J. Perez-Cruz, "Improved resolution of the MCSA method via Hilbert transform, enabling the diagnosis of rotor asymmetries at very low slip,” IEEE Trans. Energy Convers., vol. 24, no. 1, pp. 52-59, 2009.

[7] B. Xu, L. Sun, L. Xu, and G. Xu, "Improvement of the Hilbert method via esprit for detecting rotor fault in induction motors at low slip," IEEE Trans. Energy Convers., vol. 28, no. 1, pp. 225-233, 2013.

[8] A. Naha, A. K. Samanta, A. Routray, and A. K. Deb, "A method for detecting half-broken rotor bar in lightly loaded induction motors using current," IEEE Trans. Instrum. Meas., vol. 65, no. 7, pp. 1614-1625, 2016.

[9] T. Ameid, A. Menacer, H. Talhaoui, and I. Harzelli, "Broken rotor bar fault diagnosis using fast Fourier transform applied to field-oriented control induction machine: simulation and experimental study," Int. J. Adv. Manuf. Technol., vol. 92, no. 1-4, pp. 917-928, 2017.

[10] G. Singh, C. Anil Kumar, and V. N. A. Naikan, "Effectiveness of Current Envelope analysis to detect broken rotor bar and inter turn faults in an inverter fed induction motor drive," Proc. 2015 IEEE Int. Conf. Power Adv. Control Eng. ICPACE 2015, pp. 191-194, 2015.

[11] T. Wang, H. Liu, L. Zhao, J. Huang, and Z. Hou, "Quantitative broken rotor bar fault detection for closed-loop controlled induction motors," IET Electr. Power Appl., vol. 10, no. 5, pp. 403-410, 2016.

[12] Y. Maouche, M. E. K. Oumaamar, M. Boucherma, and A. Khezzar, "Instantaneous power spectrum analysis for broken bar fault detection in inverter-fed six-phase squirrel cage induction motor," Int. J. Electr. Power Energy Syst., vol. 62, pp. 110-117, 2014.

[13] T. Goktas, "Discerning broken rotor bar failure from low-frequency load torque oscillation in DTC induction motor drives," 2016.

[14] Z. Hou, J. Huang, H. Liu, M. Ye, Z. Liu, and J. Yang, "Diagnosis of broken rotor bar fault in open- and closed-loop controlled wye-connected induction motors using zero-sequence voltage," vol. 11, pp. 1214-1223, 2017.

[15] Y. Gritli, A. O. Di Tommaso, R. Miceli, F. Filippetti, and C. Rossi, "Closed-loop bandwidth impact on MVSA for rotor broken bar diagnosis in IRFOC double squirrel cage induction motor drives," 4th Int. Conf. Clean Electr. Power Renew. Energy Resour. Impact, ICCEP 2013, pp. 529-534, 2013.

[16] M. Akar and H. S. Gercekcioglu, "Instantaneous power factor signature analysis for efficient fault diagnosis in inverter fed three phased induction motors," Int. J. Hydrogen Energy, vol. 42, no. 12, pp. 8338-8345, 2017.

[17] A. M. da Silva, R. J. Povinelli, and N. A. O. Demerdash, "Induction machine broken bar and stator short-circuit fault diagnostics based on three-phase stator current envelopes," IEEE Trans. Ind. Electron., vol. 55, no. 3, pp. 1310-1318, 2008. 
[18] I. Ouachtouk, S. El, S. Guedira, K. Dahi, and H. Mediouni, "Broken Rotor Bar fault detection based on Stator Current Envelopes Analysis in Squirrel Cage Induction Machine," pp. 2-7, 2017.

[19] P. Taylor, H. Arabaci, and O. Bilgin, "A Simplified Algorithm to Detect Rotor Bar Faults by Using Stator Current Envelope A Simplified Algorithm to Detect Rotor Bar Faults by Using Stator Current Envelope,” no. December, pp. 37-41, 2014.

[20] M. Abd-el-Malek, A. K. Abdelsalam, and O. E. Hassan, "Induction motor broken rotor bar fault location detection through envelope analysis of start-up current using Hilbert transform," Mech. Syst. Signal Process., vol. 93, pp. 332-350, 2017.

[21] P. Shi, Z. Chen, Y. Vagapov, and Z. Zouaoui, "A new diagnosis of broken rotor bar fault extent in three phase squirrel cage induction motor," Mech. Syst. Signal Process., vol. 42, no. 1-2, pp. 388-403, 2014.

[22] C. Kral, F. Pirker, G. Pascoli, and H. Kapeller, "Robust rotor fault detection by means of the Vienna Monitoring Method and a parameter tracking technique," IEEE Trans. Ind. Electron., vol. 55, no. 12, pp. 4229-4237, 2008.

[23] A. Ammar, A. Bourek, and A. Benakcha, "Nonlinear SVM-DTC for induction motor drive using input-output feedback linearization and high order sliding mode control," ISA Trans., vol. 67, pp. 428-442, 2017.

[24] L. Cristaldi, A. Monti, and F. Ponci, "Three-phase Load Signature : a wavelet-based approach to power analysis," Sixth Int. Work. Power Defin. Meas. under Non-Sinusoidal Cond., 2003.

[25] S. M. A. Cruz, A. Stefani, F. Filippetti, and A. J. M. Cardoso, "A new model-based technique for the diagnosis of rotor faults in RFOC induction motor drives," IEEE Trans. Ind. Electron., vol. 55, no. 12, pp. 4218-4228, 2008.

\section{BIOGRAPHIES OF AUTHORS}
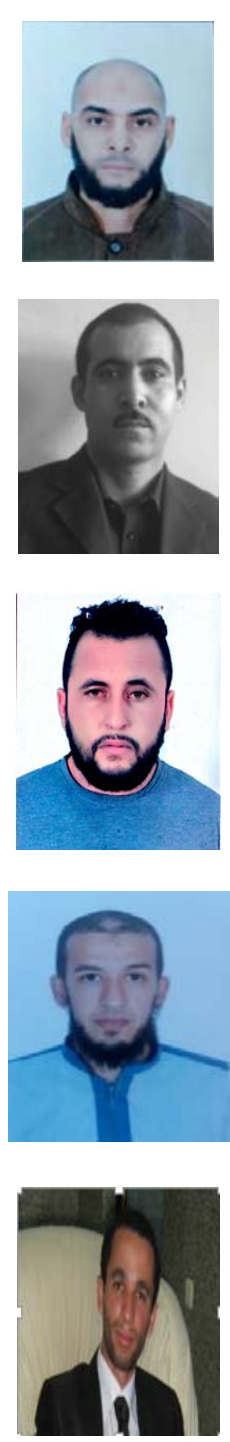

Lachtar Salah received the Engineer's degree and the Magister degree in Electric control respectively in 2003 and 2007 from Annaba University of Algeria. In 2016, he prepared his Ph.D. Thesis in control and analysis electric systems failure at Biskra University, Algeria. He joined the Research Unit on Renewable Energy in Middle-Saharan Adrar in 2013. His current research interests include control and analysis electric systems performance and failure,

Adel Ghoggal received the Engineer, Magister diploma and the $\mathrm{PhD}$ degree in electrical engineering from the University of Biskra, in 1996, 2005 and 2010 respectively. He is an assistant professor with the University of Biskra since 2005 and he is a member in the LGEB Laboratory. His research interests are focused on electrical machines design and fault diagnosis in power electronics systems and machines.

Koussa Khaled received the Engineer degree in Electronic control systems in 2010 and the Magister degree in Electronic control systems in 2013 from Ecole Nationale Polytechnique Oran, Algeria. In 2018, he prepared his Ph.D. Thesis in control and analysis electric systems failure at Bechar University, Algeria. He joined the Research Unit on Renewable Energy in Middle-Saharan Adrar in 2014. His current research interests include control and analysis electric systems performance and failure,

Ahmed Bouraiou received the Engineer's degree in Automatic and the Magister degree in Electric control respectively in 2007 and 2011 from Annaba University of Algeria, $\mathrm{PhD}$ degree from the Department of Electrical Engineering, National Polytechnic School of Oran in March 2018. He is a Researcher, in Research Unit on Renewable Energy in Middle-Saharan Adrar, Algeria (Electronic Systems Team, Photovoltaic Conversion Division). His current research interests include analysis of photovoltaic systems performance and failure, renewable energy and electrical systems control.

Issam Attoui was born in Annaba, Algeria, in 1985. He received the engineer's degree, magister's degree, and Ph.D. in electrical engineering from Badji Mokhtar-Annaba University, Algeria in 2007, 2009, and 2015 respectively. In 2011, he joined the Research Center in Industrial Technolo-gies/Research Unit in Advanced Materials (URMA/CRTI) Annaba, Algeria. His research interests include system mod- eling and control, process fault diagnosis, signal processing, renewable energy, vibration monitoring, fractional order control, and neural networks. 